\title{
Lung Transplantation in a Multidrug-Resistant Gram-Negative Acinetobacter Baumannii- Colonized Patient: A Case Report
}

\author{
Katharina Huenges ${ }^{1} \quad$ Alexander Reinecke ${ }^{1} \quad$ Burkhard Bewig $^{2} \quad$ Assad Haneya $^{1} \quad$ Jochen Cremer $^{1}$ \\ ${ }^{1}$ Department of Cardiovascular Surgery, University of Schleswig- \\ Holstein Campus Kiel, Kiel, Germany \\ 2 Department of Internal Medicine I, University of Schleswig Holstein \\ Campus Kiel, Kiel, Germany \\ Address for correspondence Katharina Huenges, MD, Department of \\ Cardiovascular Surgery, University of Schleswig-Holstein Campus Kiel, \\ Arnold-Heller-Straße 3, Hs 18, Kiel 24105, Germany \\ (e-mail: Katharina.Huenges@uksh.de).
}

Thorac Cardiovasc Surg Rep 2016;5:16-17.

\author{
Abstract \\ Keywords \\ - lung transplantation \\ - $\alpha-1$-antitrypsin \\ deficiency \\ - Multidrug-resistant \\ Acinetobacter \\ baumannii
}

Colonization or infection with various pathogens is frequently found in patients listed for lung transplantation. We describe a case of a 50 -year-old woman with $\alpha$-1-antitrypsin deficiency, which was listed for double-lung transplantation, with multidrug-resistant gram-negative Acinetobacter baumannii (MRGN4-Ab) skin colonization. Transplantation was successfully performed. Colistin (Polymyxine E) was administered intravenously and through inhalation in the first month. MRGN4-Ab was still detectable in skin swabs without evidence of infection. After good recovery and clinical inapparence, the patient was discharged 2 months after transplantation.

\section{Introduction}

In the past years, incidence of multiresistant pathogens increased, aggravating pre- and posttransplantation management and outcome of patients waiting for life-saving organ transplantation or even prohibiting transplantation. ${ }^{1,2}$

Multidrug-resistant gram-negative Acinetobacter baumannii (MRGN4- $A b$ ) is defined as acquired nonsusceptibility to at least one agent in three or more antimicrobial categories. ${ }^{2}$ So far, little is known about preoperative colonization or infection with $A$. baumannii, notably if multiresistant and/or carbapenem resistant; therefore, it has an unclear influence on transplantation outcome. ${ }^{3}$ Most case reports published to date addressed to $A$. baumannii infections are related to posttransplantation-acquired infections, including donorrecipient transmission, often associated with severe courses and high mortality. ${ }^{3-8}$

In this report, the pre- and postoperative management of a 50 -year-old patient with $\alpha$-1-antitrypsin deficiency, who was listed for double-lung transplantation, with MRGN4-Ab skin colonization is presented.

received

July 26, 2015

accepted

October 19, 2015

published online

December 16, 2015 10.1055/s-0035-1569993. ISSN 2194-7635.

\section{Case Description}

A 50-year-old woman, with a long history of pulmonary disease due to $\alpha$-1-antitrypsin deficiency and chronic pulmonic obstruction, was admitted to the hospital with respiratory failure caused by acute infectious exacerbation. The patient was listed for double-lung transplantation before. Hypercapnia necessitated admittance to intensive care unit (ICU) for passive interventional lung assist (iLA) therapy and high-flow ventilation. After 2 weeks, the patient was stabilized; iLA and mechanical ventilation could be weaned. Primarily improved clinical condition impaired again and the patient was readmitted to ICU and iLA therapy was restarted.

By this time, outbreak of MRGN4-Ab was detected at our hospital. Routine screening swabs revealed skin colonization with MRGN4-Ab in our patient. Antimicrobial susceptibility was determined; MRGN4- $A b$ isolates were susceptible to colistin (polymyxin E), intermediate to carbapenems, and resistant to all other tested antimicrobial agents. Dealing with colonization or infections of such multidrug-resistant pathogens before transplantation remained unclear, and

\footnotetext{
(c) 2016 Georg Thieme Verlag KG Stuttgart · New York
}

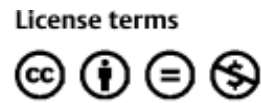


literature revealed no groundbreaking decision support. Therefore, this issue was extensively discussed in our interdisciplinary transplant conference and with official national and international institutions (i.e., medical societies, Eurotransplant, German Organ Transplantation Foundation, German Medical Association). Ultimately, the colonization was not considered as a contraindication for lung transplantation, since there were no signs for infection with this multidrug-resistant pathogen and considering the patient's worsening clinical situation.

The patient could successfully be double-lung transplanted. The initial need for further assisted ventilation or oxygen insufflation could be weaned off in the first month after transplantation. Colistin was administered intravenously (3 Mio IE three times a day) and through inhalation (1 Mio IE twice a day) in first month after transplantation and was combined with a carbapenem, like previously reported for MRGN4- $A b$ infections after transplantation. ${ }^{9}$ Immunosuppressive therapy was based on a triple-drug regimen, namely, a calcineurin inhibitor (cyclosporine), mycophenolate mofetil, and a corticosteroid according to our institutional standard protocol. Inflammatory markers remained low and there were no signs for organ rejection. Initial required hemodialysis could be stopped after colistin therapy was discontinued and re-improvement of renal function.

MRGN4- $A b$ was once detectable in catheter urine probe, therefore additional Colistin was administered intravesically. MRGN4- $A b$ was still detectable in skin swabs without evidence of systemic infection. After good recovery, adequate wound healing, and clinically inapparence, the patient could be moved to a transplant-specialized rehab clinic 2 months after transplantation.

\section{Conclusion}

Colonization or infection with various pathogens can frequently be found in patients listed for lung transplantation. Known risk factors are long-term admittance to ICU, immunosuppression, mechanical ventilation, and multiple applications of broad-spectrum antibiotics. ${ }^{3}$ Patients on the waiting list prior to transplantation are also often hospitalized and are thus at high risk for nosocomial infections. So far, little is known about regimen and outcome of multidrugresistant colonization or infection in patients requiring solid organ transplantation.

Nosocomial outbreaks of $A$. baumannii is an emerging cause for health care-associated infections. ${ }^{1}$ The most common site of $A$. baumannii infections after solid and nonsolid organ transplantation in the respiratory tract. ${ }^{4,5}$ Optimal treatment regimens are so far unknown and limited due to multidrug resistance. Appropriate antimicrobial therapy therefore can lower mortality even in transplanted patients. ${ }^{8}$ Manifestation of resistance pattern influences therapeutic regimens and outcome of the patients. Carbapenem resistance was not associated with a higher mortality in liver- and kidney-transplanted patients. ${ }^{8}$ Beside one case report of a heart transplant recipient, whereas $A$. baumannii infection was detected 4 days after transplantation in a recipient's prior collected sample, A. baumannii infections in transplantation are up to date only reported after organ transplantation. In that patient therapy contained colistin combined with teicoplanin and tigecycline. ${ }^{4}$ The patient could be discharged and was alive at the 9-month follow-up.

In our case, A. baumannii was detected 4 weeks before transplantation. The patient could successfully be transplanted. Colistin was administered intravenous and through inhalation, and was combined with a carbapenem and a standard immunosuppressive therapy. Inflammatory markers remained low and there were no signs for organ rejection.

In conclusion, our case illustrates that lung transplantation in patients colonized with multidrug-resistant pathogens can be feasible in the presence of preemptive individualized antibiotic treatment.

\section{References}

1 Fournier PE, Richet $\mathrm{H}$. The epidemiology and control of Acinetobacter baumannii in health care facilities. Clin Infect Dis 2006; 42(5):692-699

2 Magiorakos AP, Srinivasan A, Carey RB, et al. Multidrug-resistant, extensively drug-resistant and pandrug-resistant bacteria: an international expert proposal for interim standard definitions for acquired resistance. Clin Microbiol Infect 2012;18(3): 268-281

3 Martins N, Martins IS, de Freitas WV, et al. Severe infection in a lung transplant recipient caused by donor-transmitted carbapenem-resistant Acinetobacter baumannii. Transpl Infect Dis 2012; 14(3):316-320

4 Andini R, Agrusta F, Mattucci I, et al. Recipient-born bloodstream infection due to extensively drug-resistant Acinetobacter baumannii after emergency heart transplant: report of a case and review of the literature. Infection 2015;43(5):609-613

5 Kim SB, Min YH, Cheong JW, et al. Incidence and risk factors for carbapenem- and multidrug-resistant Acinetobacter baumannii bacteremia in hematopoietic stem cell transplantation recipients. Scand J Infect Dis 2014;46(2):81-88

6 Kitazono H, Rog D, Grim SA, Clark NM, Reid GE. Acinetobacter baumannii infection in solid organ transplant recipients. Clin Transplant 2015;29(3):227-232

7 Nunley DR, Bauldoff GS, Mangino JE, Pope-Harman AL. Mortality associated with Acinetobacter baumannii infections experienced by lung transplant recipients. Lung 2010;188(5):381-385

8 de Gouvêa EF, Martins IS, Halpern M, et al. The influence of carbapenem resistance on mortality in solid organ transplant recipients with Acinetobacter baumannii infection. BMC Infect Dis 2012;12:351

9 Shields RK, Clancy CJ, Gillis LM, et al. Epidemiology, clinical characteristics and outcomes of extensively drug-resistant Acinetobacter baumannii infections among solid organ transplant recipients. PLoS ONE 2012;7(12):e52349 